ARTICLE

\title{
Water cluster in hydrophobic crystalline porous covalent organic frameworks
}

\author{
Ke Tian Tan (10 ${ }^{1}$, Shanshan Tao ${ }^{1}$, Ning Huang (iD $^{1} \&$ Donglin Jiang (iD) ${ }^{1 凶}$
}

Progress over the past decades in water confinement has generated a variety of polymers and porous materials. However, most studies are based on a preconception that small hydrophobic pores eventually repulse water molecules, which precludes the exploration of hydrophobic microporous materials for water confinement. Here, we demonstrate water confinement across hydrophobic microporous channels in crystalline covalent organic frameworks. The frameworks are designed to constitute dense, aligned and one-dimensional polygonal channels that are open and accessible to water molecules. The hydrophobic microporous frameworks achieve full occupation of pores by water via synergistic nucleation and capillary condensation and deliver quick water exchange at low pressures. Water confinement experiments with large-pore frameworks pinpoint thresholds of pore size where confinement becomes dominated by high uptake pressure and large exchange hysteresis. Our results reveal a platform based on microporous hydrophobic covalent organic frameworks for water confinement.

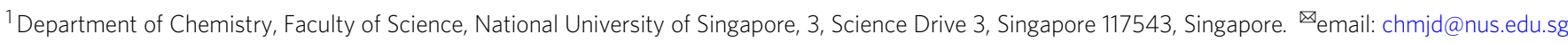


W ater confinement is ubiquitous and it plays vital roles in geological, biological and artificial systems with many applications ${ }^{1-8}$. To develop water confinement systems, extensive studies have been conducted over the past 60 years on a variety of hydrogel polymers with various hydrophilic backbones and different functionalities ${ }^{1-3}$. However, the lack of mechanistic strength and immobile water state have precluded their real implementation in water uptake and transport. To create porous structures, researchers have relied on bottom-up strategy via self-assembly of block polymers ${ }^{9-11}$. However, it is difficult to prepare stable and well-defined channels from phaseseparated polymers. In recent years, a variety of porous polymers bearing intrinsic porosity has been studied extensively for water confinement and uptake ${ }^{4-6}$. Nevertheless, the design of hydrophobic small-pore materials is precluded by a preconception that hydrophobic small pores would eventually repulse water molecules and cannot enable water confinement and uptake.

We anticipate hydrophobic supermicroporous covalent organic frameworks (COFs) with compact pore void is indispensable for water cluster occupation. COFs as a crystalline porous framework material, can be constructed via bottom-up polymerization through topological design. Upon judicious selection of building blocks, aromatic components can connect via covalent bonds to grant extended crystalline structure and hydrolytic stability in COFs. Particularly, simplistic design can generate permanent supermicroporous voids that are implausible to collapse. These distinct features can be combined in COFs and are highly desired for supermicropores but hardly accessible to other porous analogues. In this work, we constitute hydrophobic microenvironment entwined with "pseudo-hydrophilicity" strips to promote water confinement at low pressure. We further dive in to investigate the impact of pore size and shape to pinpoint the size threshold and shape for favourable water molecules confinement. We unexpectedly found that pore size threshold is distinctive to each pore shape in creating similar pore environment. We establish the size borderline of trigonal, hexagonal and tetragonal frameworks to eliminate the hysteretic behaviour in water uptake.

\section{Results}

Design and crystal structures of trigonal COFs. In this study, we demonstrate water confinement across hydrophobic microporous channels in crystalline and porous covalent organic frameworks (COFs). COFs are a unique class of polymers that enable the creation of dense, aligned and one-dimensional (1D) channels, with a diversity of applications ${ }^{12,13}$. The targeted COFs were constructed to possess predesigned 1D channels with different polygonal shape, pore size and wall environment. We synthesized microporous trigonal hydrophobic COFs, i.e., HFPTP-PDACOF $^{14}$, HFPTP-DMePDA-COF and HFPTP-BPDA-COF ${ }^{15}$ via condensation of 2,3,6,7,10,11-hexakis(4-formylphenyl) triphenylene (HFPTP) with 1,4-phenylenediamine (PDA), 2,5-dimethyl1,4-phenylenediamine (DMePDA) and 1,1'-biphenyl-4,4'-diamine (BPDA) under solvothermal conditions as previously reported (Fig. 1). Their chemical structures were characterised by analytic methods (Supplementary Figs. 1 and 2).

Powder X-ray diffraction (PXRD) pattern of the newly synthesized HFPTP-DMePDA-COF revealed peaks at $4.03^{\circ}$, $6.97^{\circ}, 8.12^{\circ}, 10.62^{\circ}, 11.07^{\circ}$ and $23.22^{\circ}$, which were assigned to the (100), (110), (200), (210), (300) and (001) facets, respectively (Fig. 2a, black curve). We used density-functional based tightbinding $(\mathrm{DFTB}+)$ calculations to optimise the conformation of single $2 \mathrm{D}$ polymer sheet and its configuration of stacking modes. The AA-stacking mode (Fig. 2a, red curve) reproduces the PXRD peak position and intensity. The HFPTP-DMePDA-COF adopts a $P_{3}$ space group of with unit-cell parameters of $a=b=25.8092 \AA$, $c=4.5279 \AA, \alpha=\beta=90^{\circ}$ and $\gamma=120^{\circ}$ (for atomistic coordinates, see Supplementary Table 1). We conducted Pawley refinements (Fig. 2a, green curve) and confirmed the correctness of the PXRD peak assignments, as indicated by a negligible difference (Fig. 2a, yellow curve) with $R_{\mathrm{wp}}=3.72 \%$ and $R_{\mathrm{p}}=$ $2.15 \%$. Rietveld refinement also yields a $P_{3}$ space group. The presence of (001) facet at $23.22^{\circ}$ (Fig. 2a, inset) suggests an extended structural ordering with an interlayer interval of $3.83 \AA$ along the $z$ direction. As the trigonal topology offers the smallest pores among COFs, HFPTP-DMePDA-COF consists of dense, aligned and microporous triangular 1D channels (Fig. 1c). On the other hand, HFPTP-PDA-COF (Fig. 2b, red curve) and HFPTPBPDA-COF (Fig. 2b, blue curve) displayed the same PXRD patterns as reported ${ }^{14,15}$, adopting AA stacking structures to form aligned microporous $1 \mathrm{D}$ channels (Fig. $1 \mathrm{a}, \mathrm{b}$ and $\mathrm{d}$ ). With the AA stacking structure, the trigonal COFs develop hydrophobic microporous channels in which dense $\mathrm{C}-\mathrm{H}$ sites are extruded from each pore wall to form an extended inner 'carpet' while the $\mathrm{C}=\mathrm{N}$ linkages are polarisable to spot "hydrophilic" sites owing to their partially charged dipole moments.

Porosity of trigonal COFs. These trigonal COFs exhibited typical type I nitrogen sorption isotherms, manifesting their microporous features (Fig. 2c, e and g). The HFPTP-PDA-COF and HFPTPDMePDA-COF exhibited a Brunauer-Emmett-Teller (BET) surface area of 662 and $480 \mathrm{~m}^{2} \mathrm{~g}^{-1}$, a pore volume of 0.32 and $0.24 \mathrm{~cm}^{3} \mathrm{~g}^{-1}$, respectively (Fig. $2 \mathrm{~d}$ and $\mathrm{f}$, Supplementary Table 2). They exhibited pore sizes of 1.1 and $1.4 \mathrm{~nm}$ (Fig. $2 \mathrm{~d}$ and f), which are consistent with their crystal structures. On the other hand, HFPTP-BPDA-COF exhibited a BET surface area of $758 \mathrm{~m}^{2} \mathrm{~g}^{-1}$, a pore volume of $0.39 \mathrm{~cm}^{3} \mathrm{~g}^{-1}$ and pore sizes of 1.2 and $1.6 \mathrm{~nm}$ (Fig. $2 \mathrm{~g}$ and $\mathrm{h}$ ). Noticeably, the cumulative pore size distribution profiles revealed that the contribution of $1.1-$ or $1.2-\mathrm{nm}$ pore is significantly larger than that of $1.4-$ or $1.6-\mathrm{nm}$ pore, indicating that the small-size supermicropore predominates the materials (Fig. 2d, $\mathrm{f}$ and $\mathrm{h}$ ). Compared to HFPTP-PDA-COF and HFPTPDMePDA-COF, HFPTP-BPDA-COF has a 0.1-nm larger pore size. Minor large pores at $1.8,4.1$ and $3.8 \mathrm{~nm}$ are observed in HFPTP-PDA-COF, HFPTP-DMePDA-COF and HFPTP-BPDA$\mathrm{COF}$ due to an incomplete connection between neighbouring pores. ${ }^{15}$ Nevertheless, these pores occupy a negligible percentage in terms of pore volume and thus do not impact the water sorption behaviour.

Water sorption and confinement of trigonal COFs. The water vapour sorption isotherms of HFPTP-PDA-COF (Fig. 3a-c, red dots and curves) resemble a typical $S$-shaped type-V sorption curve, which is characteristic of hydrophobic materials ${ }^{16-18}$. The HFPTP-PDA-COF at $25^{\circ} \mathrm{C}$ (Fig. 3a, red dots and curve) exhibited an induction pressure zone $\left(\mathrm{P} / \mathrm{P}_{0}\right)$ of $0-0.4$, where water molecules are nucleated in the small channel. After the induction zone, a steep water uptake occurs at a low $\mathrm{P} / \mathrm{P}_{0}$ of 0.4 and completes at $\mathrm{P} / \mathrm{P}_{0}$ of 0.41 . The quick uptake originates from capillary condensation of water molecules in the microporous $1 \mathrm{D}$ channels. Similarly, HFPTP-PDA-COF at $15^{\circ} \mathrm{C}$ and $10^{\circ} \mathrm{C}$ (Fig. $3 \mathrm{~b}$ and $\mathrm{c}$, red dots and curve) displayed an induction $\mathrm{P} / \mathrm{P}_{0}$ zone of $0-0.37,0-0.38$ and a sharp capillary condensation at $\mathrm{P} / \mathrm{P}_{0}$ of $0.37-0.40,0.38-0.40$. Such a sharp uptake at a low pressure is attractive for water confinement. In contrast, HFPTP-DMePDACOF with the same pore size but bearing methyl groups on pore walls totally changes the behaviour. The HFPTP-DMePDA-COF exhibited an induction $\mathrm{P} / \mathrm{P}_{0}$ zone of $0-0.65$ at $25^{\circ} \mathrm{C}$ (Fig. 3a, blue dots and curve), $0-0.63$ at $15^{\circ} \mathrm{C}$ (Fig. $3 \mathrm{~b}$, blue dots and curve) and $0-0.61$ at $10^{\circ} \mathrm{C}$ (Fig. $3 \mathrm{c}$, blue dots and curve), which are much far broader than those of HFPTP-PDA-COF. More significantly, 
a
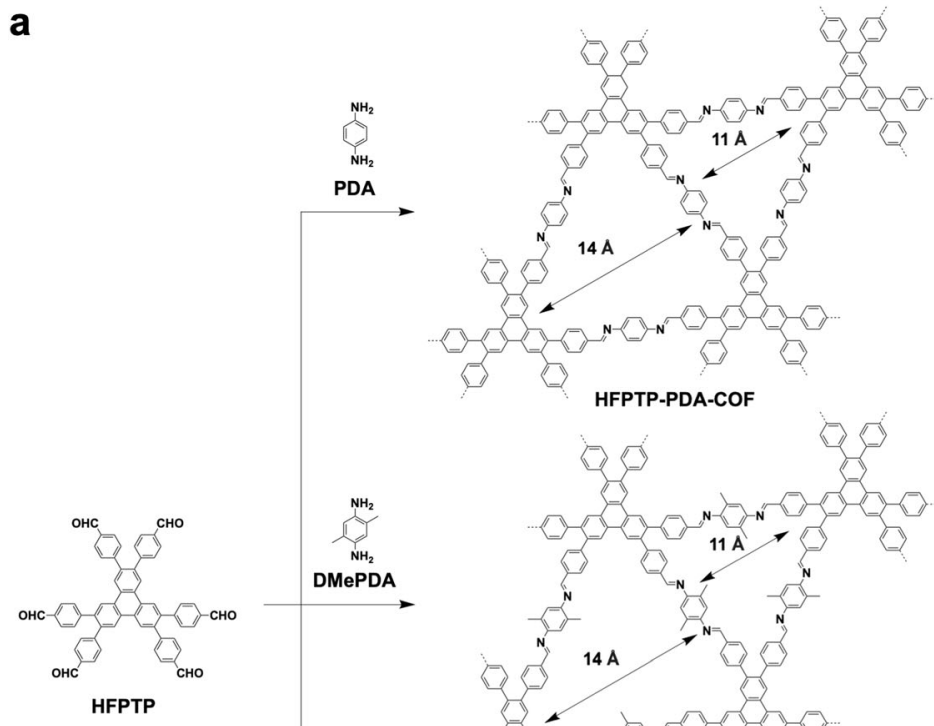

HFPTP-PDA-COF

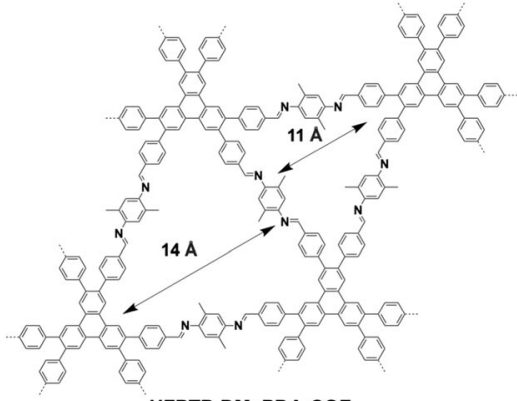

HFPTP-DMEPDA-COF

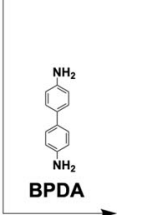

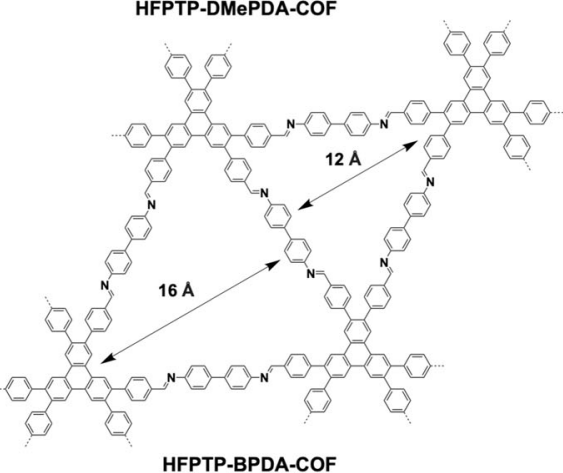
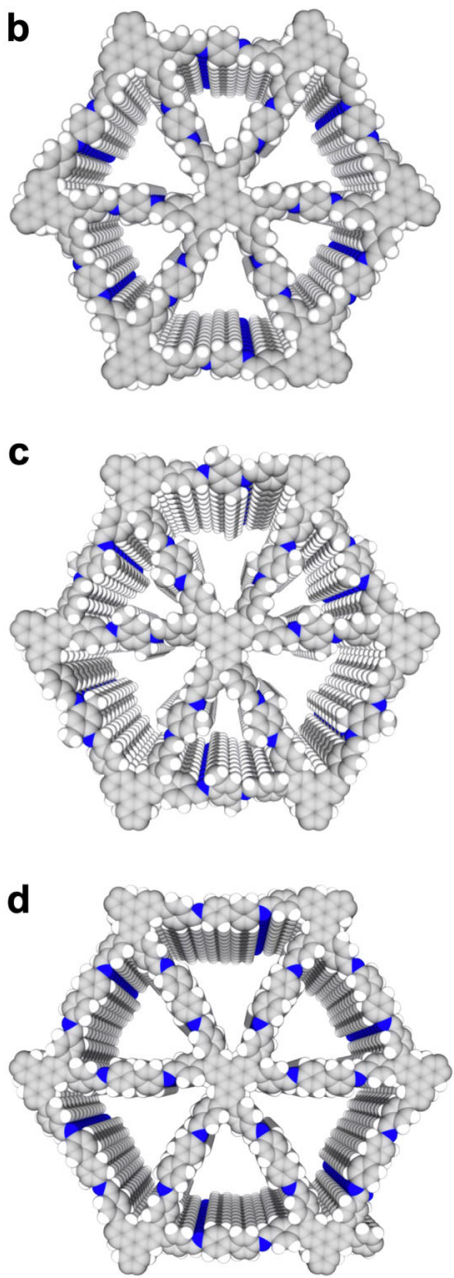

Fig. 1 Hydrophobic supermicroporous 1D channels in trigonal COFs. a Schematic of synthesis of HFPTP-PDA-COF, HFPTP-DMePDA-COF and HFPTPBPDA-COF. Reconstructed structures of (b) HFPTP-PDA-COF, (c) HFPTP-DMePDA-COF and (d) HFPTP-BPDA-COF.

HFPTP-DMePDA-COF did not show a steep uptake but displayed a rather sluggish adsorption over a higher and broader $\mathrm{P} /$ $\mathrm{P}_{0}$ range of $0.6-0.9$ at $25^{\circ} \mathrm{C}, 0.6-0.9$ at $15^{\circ} \mathrm{C}$ and $0.6-0.8$ at $10^{\circ} \mathrm{C}$, respectively. These drastic changes reflect that a small perturbation of the pore walls completely alters the water confinement in the hydrophobic triangular supermicropores.

The HFPTP-BPDA-COF with a pore size of $1.2 \mathrm{~nm}$ exhibited an induction $\mathrm{P} / \mathrm{P}_{0}$ zone of $0-0.52$ at $25^{\circ} \mathrm{C}$ (Fig. 3a, black dots and curve), $0-0.51$ at $15^{\circ} \mathrm{C}$ (Fig. $3 \mathrm{~b}$, black trigons and curve) and $0-0.50$ at $10^{\circ} \mathrm{C}$ (Fig. $3 \mathrm{c}$, black squares and curve), respectively, which are much broader than those of HFPTP-PDA-COF with a pore size of $1.1 \mathrm{~nm}$ (Fig. $3 \mathrm{a}-\mathrm{c}$, red symbols and curves). Capillary condensation was observed but it occurred at much higher pressures of $0.52-0.58$ at $25^{\circ} \mathrm{C}, 0.52-0.57$ at $15^{\circ} \mathrm{C}$ and $0.50-0.58$ at $10^{\circ} \mathrm{C}$, respectively. The greatly broadened induction zone together with a dramatic shift to higher pressure reflects that water is also highly sensitive to the pore size of triangular channels, where large-pore trigonal COFs with a pore size larger than $1.2 \mathrm{~nm}$ are no longer favourable for water confinement.

We evaluated the pore occupancy by assuming the density of water to be $0.997 \mathrm{~g} \mathrm{~cm}^{-3}$ using Eq. (1). Notably, HFPTP-PDACOF and HFPTP-BPDA-COF at $25^{\circ} \mathrm{C}$ exhibited a pore occupancy of $93 \%$ and $92 \%$, respectively, denoting that the channel void space is highly accessible to water molecules (Fig. 3d). In contrast, HFPTP-DMePDA-COF at $25^{\circ} \mathrm{C}$ displayed a pore occupancy of only $49 \%$, which is only half of those of HFPTP-PDA-COF and HFPTP-BPDA-COF. The much lower pore accessibility originates from the methyl units on the wall surface which impose a severe steric hindrance for water access to the neighbouring hydrophilic $\mathrm{N}$ chains. This spatial restriction extends along the $z$ direction on each pore walls and causes difficulty of water cluster formation to reach a high pore filling.

$$
\text { Pore occupancy }=\frac{\text { Uptake capacity }}{\text { Pore volume }} \times 100 \%
$$

Regime of hexagonal and tetragonal COFs. With the distinct pore size and environment thresholds in trigonal COFs, we further investigated if pore shape would trigger different confinement property (Fig. 4a-h, Supplementary Table 3). COFs are unique to enable the predesign of $1 \mathrm{D}$ channels with different polygonal shapes and sizes while retaining the same pore wall structures; we thus synthesised microporous tetragonal TFBCzPDA-COF ${ }^{19}$ (Fig. 4a, pore size $=1.5 \mathrm{~nm}$, Supplementary Fig. 3a-c), microporous hexagonal TTA-TFB-COF 20 (Fig. 4b, pore size $=1.5 \mathrm{~nm}$, Supplementary Fig. $3 \mathrm{~d}-\mathrm{f}$ ), mesoporous tetragonal TFPPy-PDA-COF ${ }^{21}$ (Fig. $4 \mathrm{c}$, pore size $=2.1 \mathrm{~nm}$, Supplementary Fig. $4 \mathrm{a}-\mathrm{c}$ ) and mesoporous hexagonal TPB-DMTP$\mathrm{COF}^{22}$ (Fig. $4 \mathrm{~d}$, pore size $=3.2 \mathrm{~nm}$, Supplementary Fig. $4 \mathrm{~d}-\mathrm{f}$ ). These COFs create dense and aligned 1D open channels, similar to the trigonal COFs in terms of hydrophobic $\mathrm{C}-\mathrm{H}$ "carpet" and "hydrophilic" $\mathrm{C}=\mathrm{N}$ sites on walls (Fig. 4e-h).

Microporous tetragonal TFBCz-PDA-COF (Supplementary Fig. 5a and c) and hexagonal TTA-TFB-COF (Supplementary Fig. 5a and d) 
a
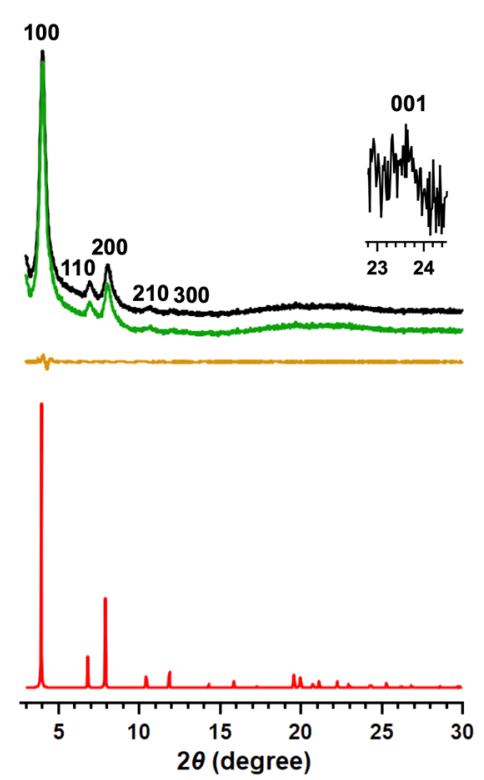

b
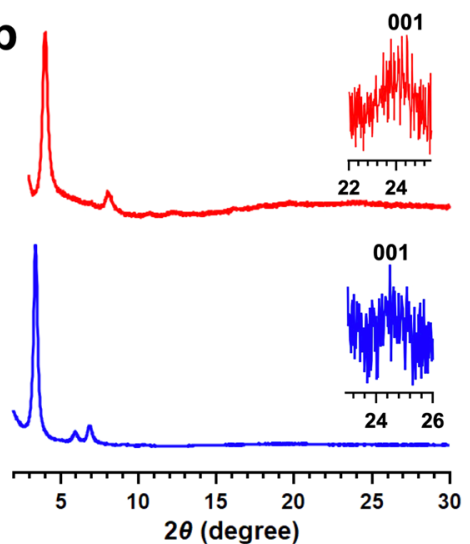

C

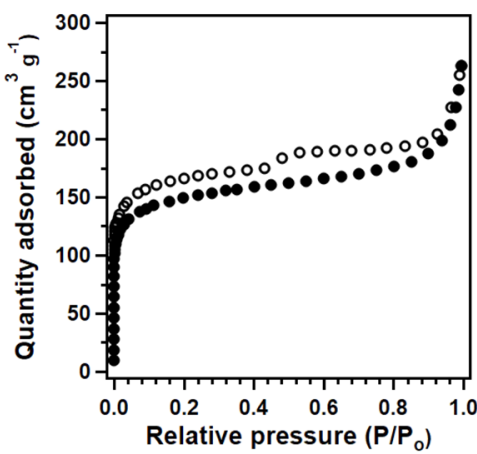

e

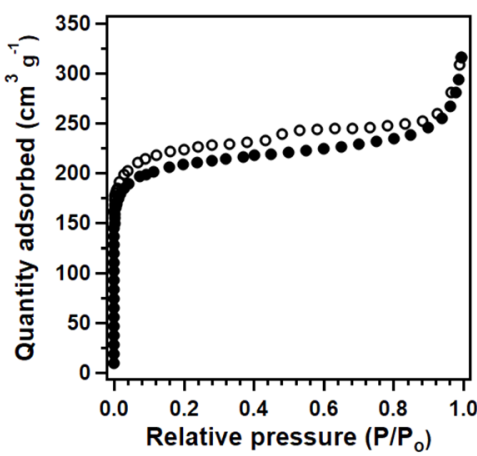

g

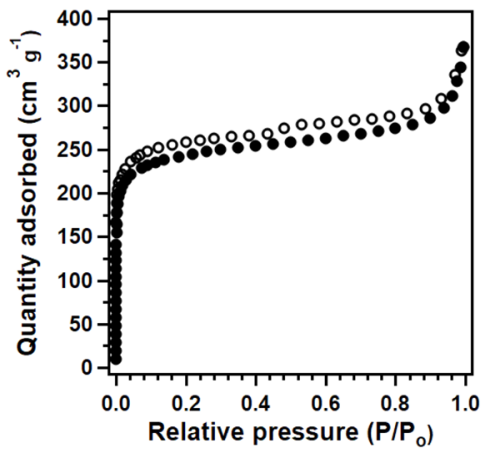

d

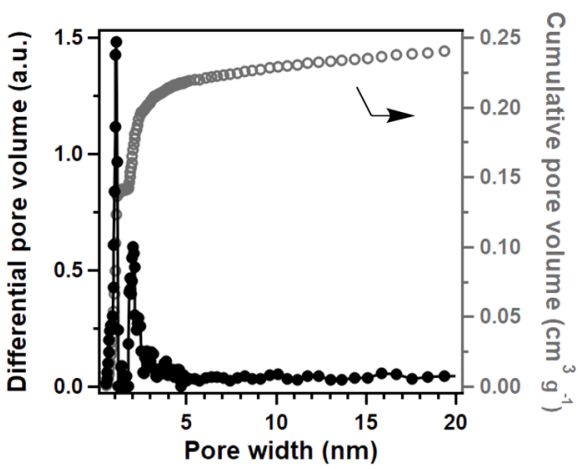

f

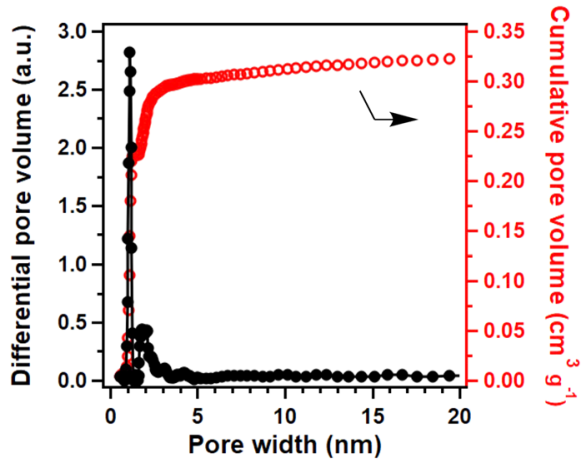

h

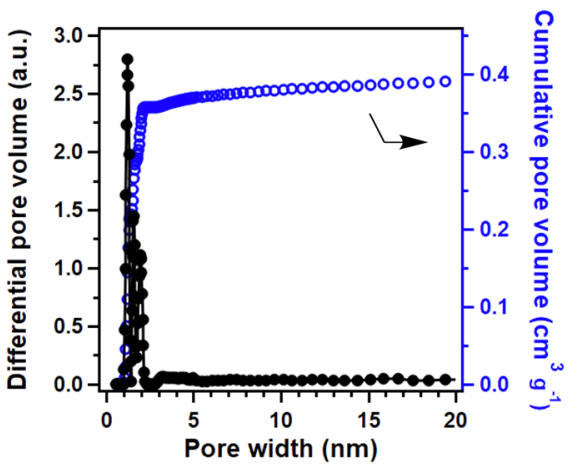

Fig. 2 PXRD and Porosity. a PXRD patterns of HFPTP-DMePDA-COF (black curve), the Pawley refinement result (green curve) and their difference (yellow curve) and the AA-stacking mode of the $P_{3}$ space group (red curve). $\mathbf{b}$ PXRD patterns of HFPTP-PDA-COF (red curve) and HFPTP-BPDA-COF (blue curve). c, Nitrogen sorption isotherm of HFPTP-DMePDA-COF. d Pore size distribution and pore volume of HFPTP-DMePDA-COF. e Nitrogen sorption isotherm of HFPTP-PDA-COF. f, Pore size distribution and pore volume of HFPTP-PDA-COF. $\mathbf{g}$ Nitrogen sorption isotherm of HFPTP-BPDA-COF. h Pore size distribution and pore volume of HFPTP-BPDA-COF.

have a pore size of $1.5 \mathrm{~nm}$ and achieve similar induction $\mathrm{P} / \mathrm{P}_{0}$ zone of $0-0.44$ at $25^{\circ} \mathrm{C}$ (Fig. $4 \mathrm{i}$, red and blue symbols and curves), $0-0.42$ at $15^{\circ} \mathrm{C}$ (Fig. $4 \mathrm{j}$, red and blue symbols and curves) and $0-0.42$ at $10^{\circ} \mathrm{C}$ (Fig. 4k, red and blue symbols and curves), respectively. Both COFs exhibited a sharp uptake at $\mathrm{P} / \mathrm{P}_{0}$ of $0.4-0.45$ owing to capillary condensation (Fig. 4i-k, red and blue symbols and curves). Noticeably, TFBCz-PDA-COF and TTA-TFB-COF at $25^{\circ} \mathrm{C}$ exhibited a pore occupancy of $79 \%$ and $97 \%$, respectively (Fig. 4l). Although tetragonal TFBCz-PDA-COF and hexagonal TTA-TFBCOF have a larger micropore $(1.5 \mathrm{~nm})$ than the trigonal HFPTPPDA-COF $(1.1 \mathrm{~nm})$, however, their induction zones and capillary condensation pressures are close to those of trigonal HFPTP-PDACOF. These results revealed that the channel shape plays an important role in water confinement and tetragonal and hexagonal COFs shift the threshold of pore size to a large micropore side. A distinct feature of water exchange in these hydrophobic micropores (adsorption and desorption) is that it occurs at a low $\mathrm{P} / \mathrm{P}_{0}$ around 0.4 within a narrow pressure window (small hysteresis loop), which is attractive for water flux and heat-pump implementation ${ }^{23}$.
Compared to microporous analogues, mesoporous tetragonal and hexagonal COFs greatly increase the uptake pressure (Fig. 4i-k, green and black symbols and curves). In the case of TFPPy-PDA-COF (pore size $=2.1 \mathrm{~nm}$ ), the induction $\mathrm{P} / \mathrm{P}_{0}$ zone was greatly broadened to $0-0.56$ at $25^{\circ} \mathrm{C}$ (Fig. $4 \mathrm{i}$, green dots and curve), $0-0.55$ at $15^{\circ} \mathrm{C}$ (Fig. $4 \mathrm{j}$, green trigons and curve) and $0-0.58$ at $10^{\circ} \mathrm{C}$ (Fig. $4 \mathrm{k}$, green squares and curve). A more explicit broadening was observed for the hexagonal large-pore TPBDMTP-COF (pore size $=3.2 \mathrm{~nm}$ ), which exhibited an induction $\mathrm{P} / \mathrm{P}_{0}$ zone of $0-0.72$ at $25^{\circ} \mathrm{C}$ (Fig. 4i, black dots and curve), $0-0.72$ at $15^{\circ} \mathrm{C}$ (Fig. 4 j, black trigons and curve) and $0-0.68$ at $10^{\circ} \mathrm{C}$ (Fig. 4k, black squares and curve), respectively. A further drawback is that the mesoporous COFs display a large hysteresis loop to complete the adsorption-desorption exchange cycle (Fig. 4i-k, black and green symbols and curves) similar to mesoporous silica FSM-16, MPS-1, MPS-2 and MPS-3 ${ }^{24,25}$. For example, TPB-DMTP-COF exhibited a hysteric loop over a wide $\mathrm{P} / \mathrm{P}_{0}$ range of $0.48-0.9$ at $25^{\circ} \mathrm{C}, 0.44-0.9$ at $15^{\circ} \mathrm{C}$ and $0.42-0.9$ at $10^{\circ} \mathrm{C}$, respectively. The $2.2-\mathrm{nm}$ pore TFPPy-PDA-COF at $25^{\circ} \mathrm{C}$ 
a

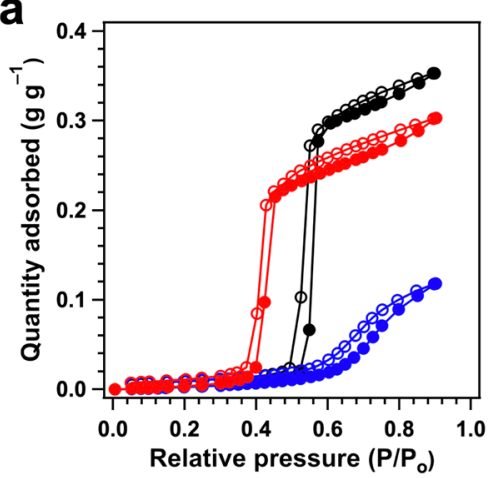

C

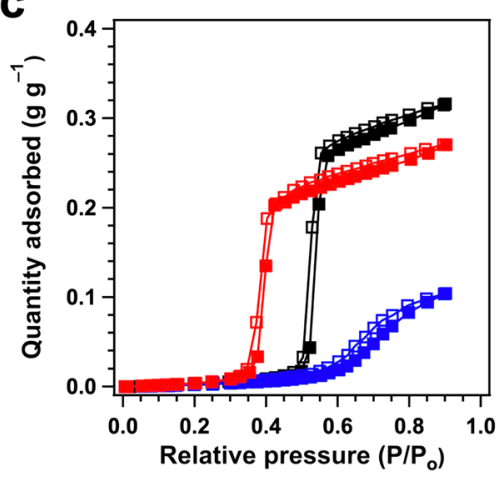

e

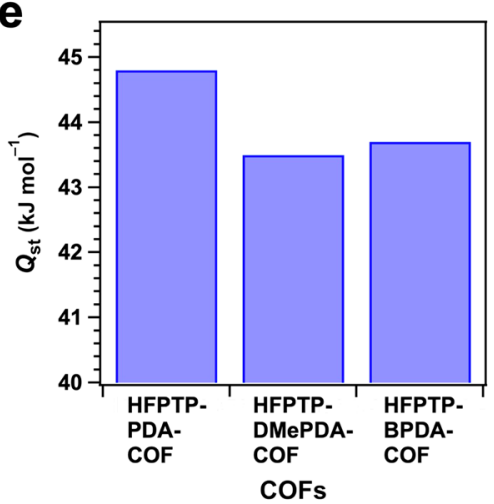

g

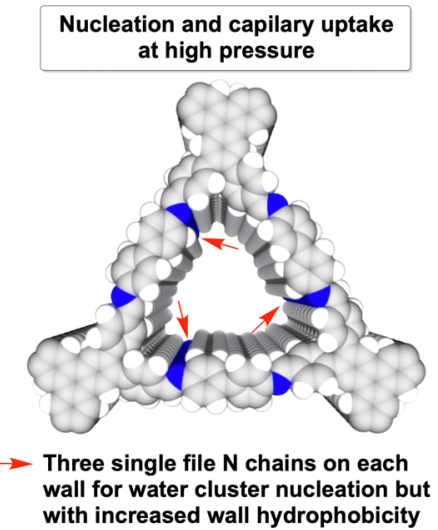

b

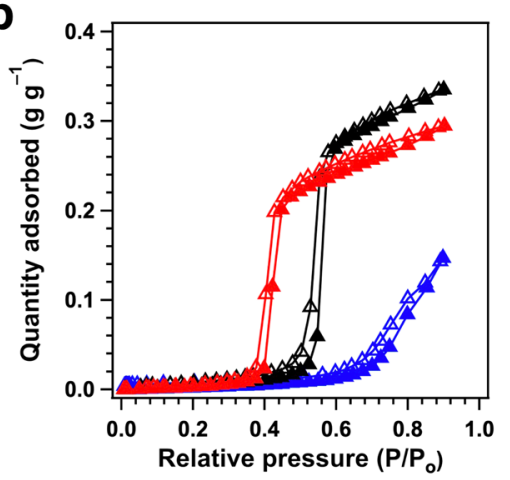

d

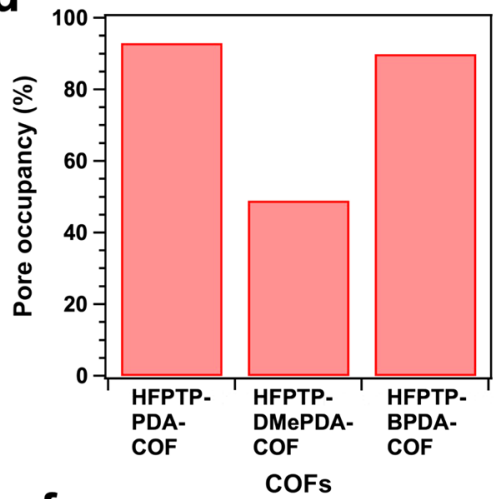

f

Nucleation and capilary uptake at low pressure

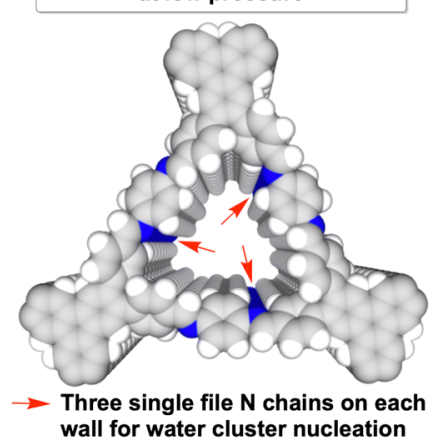

h

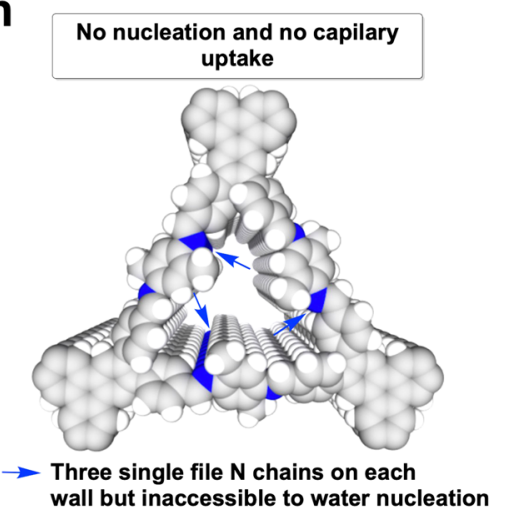

Fig. 3 Water vapour uptake and pore accessibility in trigonal COFs. a Vapour sorption isotherms of HFPTP-PDA-COF (red), HFPTP-DMePDA-COF (blue) and HFPTP-BPDA-COF (black) measured at $25^{\circ} \mathrm{C}$ (filled circle-adsorption branch, empty circle-desorption branch). $\mathbf{b}$ Vapour sorption isotherms of HFPTP-PDA-COF (red), HFPTP-DMePDA-COF (blue) and HFPTP-BPDA-COF (black) measured at $15^{\circ} \mathrm{C}$ (filled trigon - adsorption branch, empty trigon desorption branch). c Vapour sorption isotherms of HFPTP-PDA-COF (red), HFPTP-DMePDA-COF (blue) and HFPTP-BPDA-COF (black) measured at $10^{\circ} \mathrm{C}$ (filled square - adsorption branch, empty square - desorption branch). d Pore occupancies of trigonal COFs. e Isosteric heat of adsorption ( $Q_{s t}$ ) of trigonal COFs. $\mathbf{f}-\mathbf{h}$ The hydrophobic $\mathrm{C}-\mathrm{H}$ 'carpet' and 'hydrophilic' single file $\mathrm{C}=\mathrm{N}$ chains (blue chains) on walls of ( $f$ ) HFPTP-PDA-COF, ( $g$ ) HFPTP-BPDACOF and (h) HFPTP-DMePDA-COF. 
a

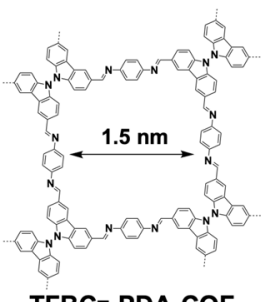

TFBCZ-PDA-COF

C

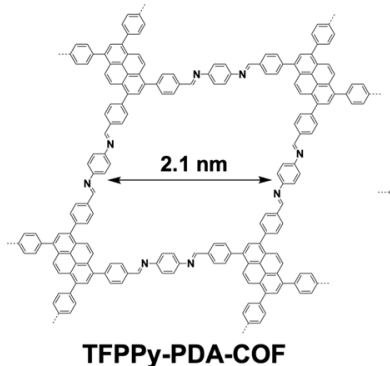

TFPPy-PDA-COF b

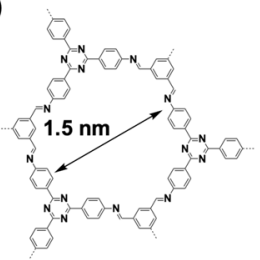

TTA-TFB-COF

d

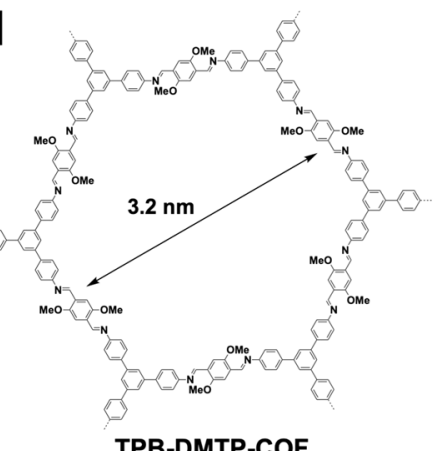

e

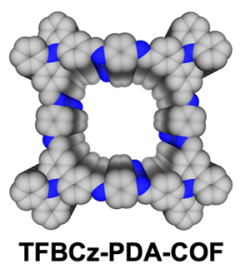

g

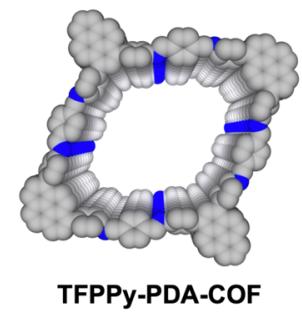

$\mathbf{f}$

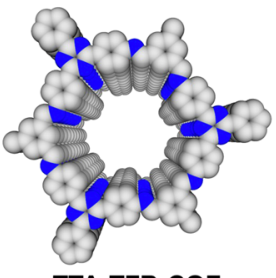

TTA-TFB-COF

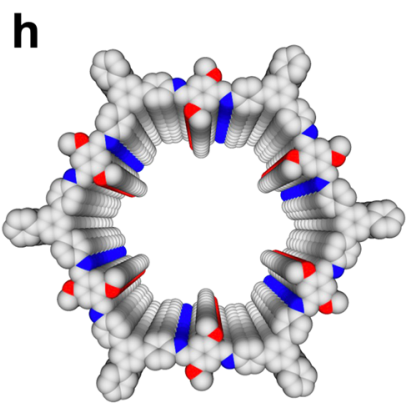

TPB-DMTP-COF
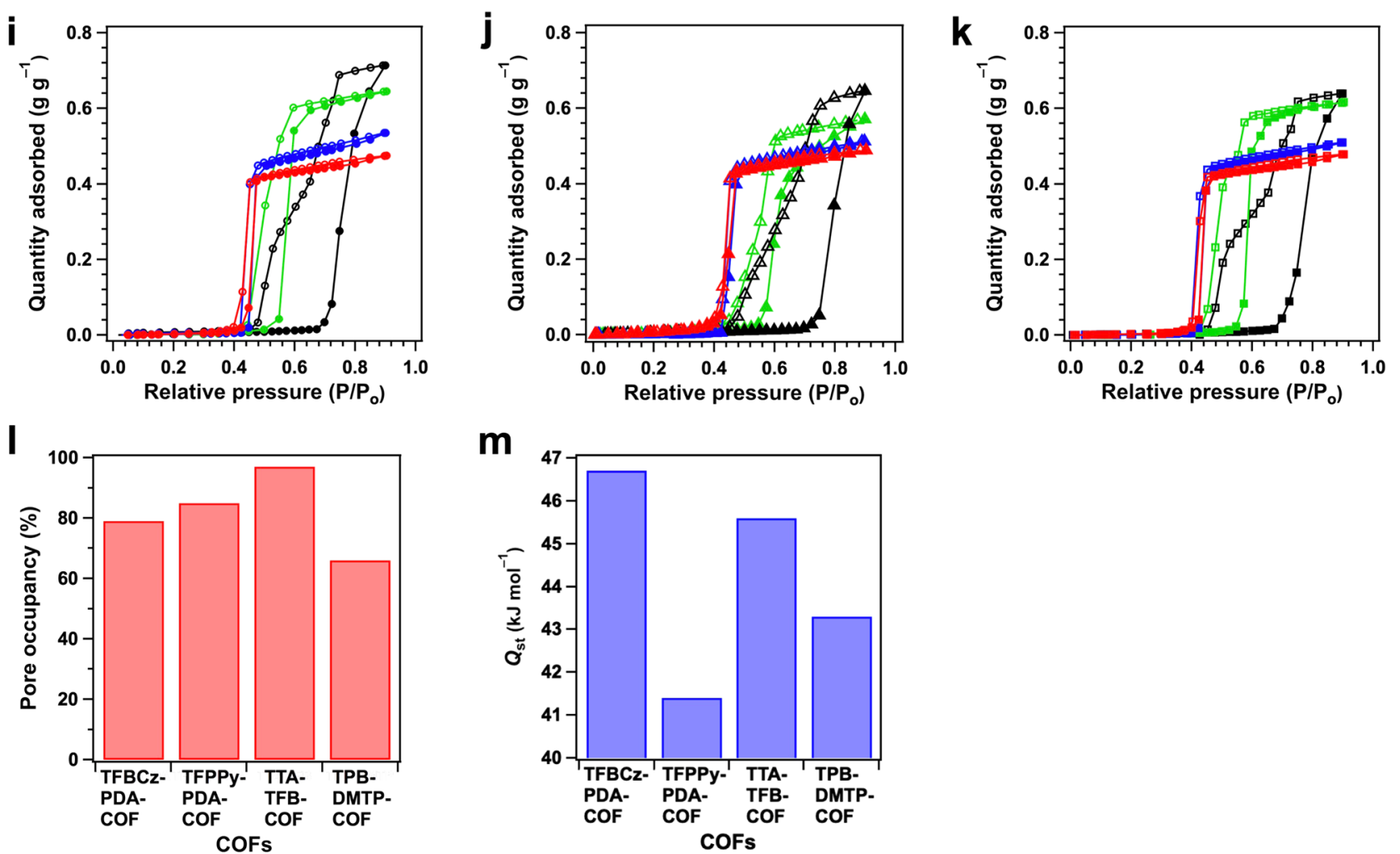

Fig. 4 Diversity of pore shape and size of hydrophobic 1D channels. a Topological synthesis of TFBCz-PDA-COF. b Topological synthesis of TTA-TFBCOF. c Topological synthesis of TFPPy-PDA-COF. $\mathbf{d}$ Topological synthesis of TPB-DMTP-COF. Reconstructed structures of (e) TFBCz-PDA-COF, (f) TTATFB-COF, (g) TFPPy-PDA-COF and (h) TPB-DMTP-COF. $\mathbf{i}$ Vapour sorption isotherms of TFBCz-PDA-COF (red), TTA-TFB-COF (blue), TFPPy-PDA-COF (green) and TPB-DMTP-COF (black) measured at $25^{\circ} \mathrm{C}$ (filled circle - adsorption branch, empty circle - desorption branch). $\mathbf{j}$ Vapour sorption isotherms of TFBCz-PDA-COF (red), TTA-TFB-COF (blue), TFPPy-PDA-COF (green) and TPB-DMTP-COF (black) measured at $15^{\circ} \mathrm{C}$ (filled trigon - adsorption branch, empty trigon - desorption branch). $\mathbf{k}$ Vapour sorption isotherms of TFBCZ-PDA-COF (red), TTA-TFB-COF (blue), TFPPy-PDA-COF (green) and TPB-DMTP-COF (black) measured at $10^{\circ} \mathrm{C}$ (filled square - adsorption branch, empty square - desorption branch). I Pore occupancy. $\mathbf{m} \mathrm{Q}_{\mathrm{st}}$ of TFBCz-PDACOF, TFPPy-PDA-COF, TTA-TFB-COF and TPB-DMTP-COF.

displayed a pore occupancy of $85 \%$ while the $3.1-\mathrm{nm}$ pore TPB-DMTP-COF dropped the pore occupancy to only $57 \%$ (Fig. 4l).

To demonstrate the importance of being $1 \mathrm{D}$ channels, we synthesised low-crystallinity TTA-TFB-CMP (Supplementary
Fig. 6) which possesses the same components as TTA-TFBCOF. Surprisingly, TTA-TFB-CMP diminishes the steep uptake and portrays only a sluggish uptake (Supplementary Fig. 6d). This result indicates that the $1 \mathrm{D}$ channel is of the utmost importance for water confinement. 
a

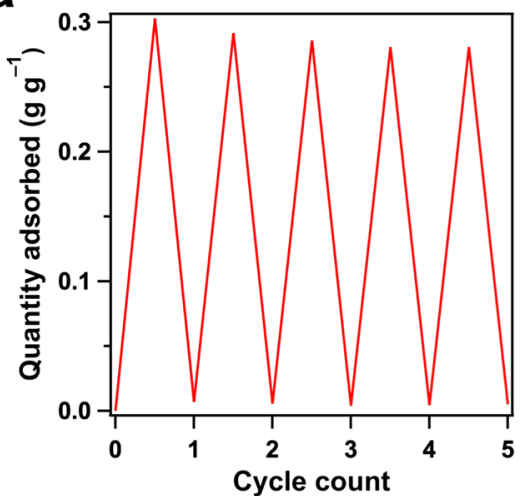

C

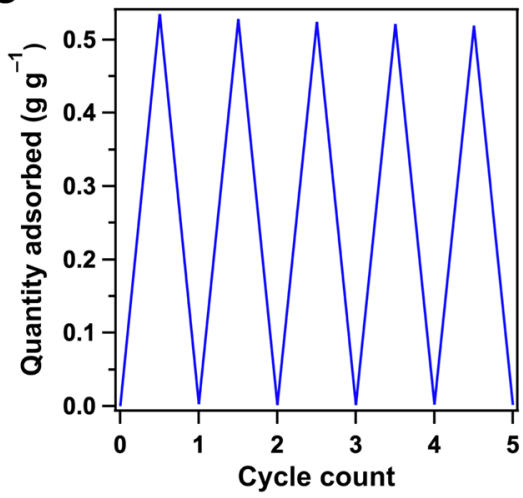

b

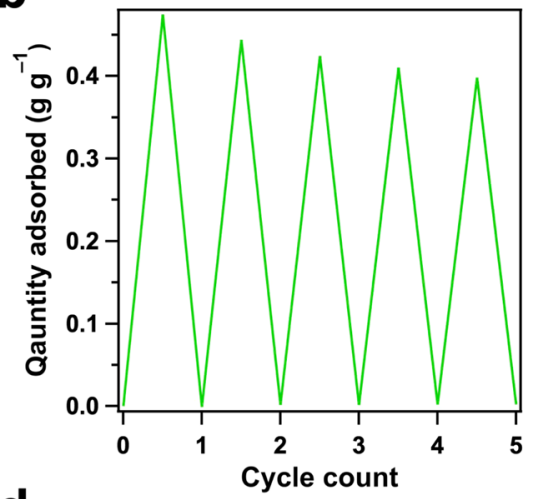

d

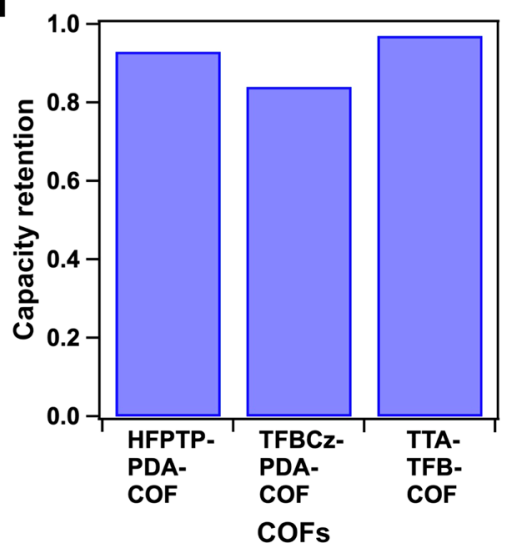

Fig. 5 Cycle performance of water confinement. a Cycle performance of HFPTP-PDA-COF. b Cycle performance of TFBCz-PDA-COF. c Cycle performance of TTA-TFB-COF. $\mathbf{d}$ Capacity retention of HFPTP-PDA-COF, TFBCz-PDA-COF and TTA-TFB-COF.

Bulk water 26 possesses heat of vaporisation of $44 \mathrm{~kJ} \mathrm{~mol}^{-1}$ at $25^{\circ} \mathrm{C}$. We investigated the isosteric heat of adsorption $\left(Q_{\mathrm{st}}\right)$ of water confinement in COFs (Supplementary Fig. 7). The HFPTPPDA-COF exhibited a $Q_{\text {st }}$ value of $44.8 \mathrm{~kJ} \mathrm{~mol}^{-1}$, while HFPTPDMePDA-COF and HFPTP-BPDA-COF displayed a $Q_{\text {st }}$ value of 43.5 and $43.7 \mathrm{~kJ} \mathrm{~mol}^{-1}$, respectively (Fig. 3e, Supplementary Table 3). The $Q_{\text {st }}$ value (Fig. $4 \mathrm{~m}$ ) of mesoporous TFPPy-PDACOF and TPB-DMTP-COF is 41.4 and $43.3 \mathrm{~kJ} \mathrm{~mol}^{-1}$, respectively, which are lower than those of supermicroporous $\mathrm{TFBCz}$ PDA-COF $\left(46.7 \mathrm{~kJ} \mathrm{~mol}^{-1}\right)$ and TTA-TFB-COF $\left(45.6 \mathrm{~kJ} \mathrm{~mol}^{-1}\right)$. These results manifest that hydrophobic microporous COFs enable stronger water confinement than mesoporous COFs (Supplementary Table 3), highlighting their high-water capture ability. By merging the feature of narrow induction pressure zone, steep uptake and high absorption heat into one material, it is safe to reach a conclusion that hydrophobic microporous channels are superb for water confinement and exchange.

\section{Discussion}

The above studies on COFs pinpoint a clear threshold of pore size, shape and environment for water confinement and exchange. In trigonal supermicroporous COFs, pore walls are densely covered with aromatic $\mathrm{C}-\mathrm{H}$ 'carpet', endowing the porous space with a high hydrophobic feature where water molecules are expected to interact with the walls weakly via van der Waals forces (Fig. $3 \mathrm{f}-\mathrm{h}$ ). On the other hand, the $\mathrm{C}=\mathrm{N}$ linkage site is polarised by the large electronegativity of nitrogen atom to form partially charged species $\left(\mathrm{C}^{\delta+}-\mathrm{N}^{\delta-}\right)$ and trigger dipolar 'hydrophilicity'. Noticeably, the polarised $\mathrm{C}=\mathrm{N}$ site is aligned vertically to form single file $\mathrm{C}^{\delta}$ ${ }^{+}-\mathrm{N}^{\delta-}$ chains, constituting a strip of 'hydrophilic' zone along the $z$ direction on each wall (Fig. 3f-h). These 'hydrophilic' strips on the hydrophobic walls offer sites for nucleation of water clusters in the channels. Such an aligned yet bicontinuous hydrophobic and 'hydrophilic' pore interface is unique in COFs and inaccessible to other porous polymers. In HFPTP-PDA-COF, at a low relative pressure $\left(\mathrm{P} / \mathrm{P}_{0}=0-0.38\right)$, the 'hydrophilic' $\mathrm{C}=\mathrm{N}$ strip serves as an anchor to enable the nucleation of water clusters (Fig. $3 \mathrm{f}$ ). As the pressure passes this threshold, the water clusters grow sharply until $0.40 \mathrm{P} / \mathrm{P}_{0}$ and instant pore filling occurs via capillary condensation $^{27}$. In contrast, HFPTP-DMePDA-COF is different in the confinement process and mechanism. The methyl groups owing to hyperconjugation effect weaken polarisation of the $\mathrm{C}=\mathrm{N}$ linkages ${ }^{28}$ to decrease the 'hydrophilicity' and spatially set a steric barrier to prevent the access to water molecules (Fig. 3h). These two-fold effects broaden the induction pressure zone and eliminate capillary condensation. In this case, water molecules are confined by sluggish diffusion via water-water intermolecular hydrogen bonds, resulting in an isotherm convex to the pressure axis. The 1.2-nm pore HFPTP-BPDA-COF with biphenyl linker constitutes much dense hydrophobic $\mathrm{C}-\mathrm{H}$ sites on the pore wall while the $\mathrm{C}=\mathrm{N}$ sites retain almost unchanged in density and are accessible to water molecules (Fig. 3g). As a result, HFPTP-PDACOF with a $0.1-\mathrm{nm}$ increased pore size greatly shifts the confinement to a much higher pressure. In the tetragonal and hexagonal microporous COFs, their pore wall environments are similar to HFPTP-PDA-COF, so that nucleation and capillary condensation occur at a low pressure. In contrast, in mesoporous tetragonal and hexagonal COFs, the wall environments become similar to HFPTP-BPDA-COF while the increased pore size likely triggers further shift to high pressure and large hysteresis loop.

The low pressure, high pore occupancy and quick water exchange cycle are attractive in heat pump implementation. We investigated cycling stability of HFPTP-PDA-COF (Fig. 5a, Supplementary Fig. 5b), TFBCz-PDA-COF (Fig. 5b, 
Supplementary Fig. 5c) and TTA-TFB-COF (Fig. 5c, Supplementary Fig. 5d). They can all retain more than $84 \%$ and even up to $97 \%$ of their maximum capacity after 5 cycles (Fig. 5 d). Robust capacity retention highlights the superiority of these COFs as heat-pump adsorbents for pro-longed cycling. Noticeably, all the microporous trigonal, tetragonal and hexagonal COFs retain their structural integrity with the identical peaks in PXRD patterns (Supplementary Figs. 8-10) and FT IR spectra (Supplementary Figs. 2, 11 and 12). The robustness against water vapour enables COFs as a suitable candidate for adsorption energy recovery and desorption cooling systems which require porous materials with high water capacity and stability ${ }^{16}$. This is superior to some metal-organic frameworks that are prone to structural degradation after continuous exposure to water ${ }^{29}$. The HFPTP-PDACOF after 5 cycles exhibited a BET surface area of $505 \mathrm{~m}^{2} \mathrm{~g}^{-1}$ and a pore volume of $0.23 \mathrm{~cm}^{3} \mathrm{~g}^{-1}$ (Supplementary Fig. 13). Noticeably, the 1.1-nm micropore is well retained while defects are selfhealed to define a flat plateau in the pore size distribution profile after cycle use (Supplementary Fig. 13c, red curve).

By developing a series of crystalline porous covalent organic frameworks, we have successfully shown water confinement in hydrophobic microporous channels. Microporous COFs are unique in that they merge high accessibility, steep uptake at low pressure, negligible hysteresis loop, large adsorption heat and robust cyclability in one material. This feature originates from the twofold effects of small pores: one is that the pore walls are covered by dense aromatic C-H 'carpet' which coat a hydrophobic inner wall; another one is that the built-in $\mathrm{C}=\mathrm{N}$ linkages polarise to afford 'hydrophilicity' of certain degree, so that their vertical alignment along the $z$ direction forms single file $\mathrm{C}=\mathrm{N}$ chain to develop a strip to enable the nucleation of water cluster. Such an ordered yet spatially separated bicontinuous structure on each pore wall renders the microporous channels able to confine water clusters quickly.

Among the microporous COFs, trigonal channel is the most promising one as it is much more sensitive to pore size and environment. A slight increase in pore size by $0.1 \mathrm{~nm}$ drastically changes the confinement while a small wall perturbation with methyl unit switches off the capillary condensation to a sluggish diffusion mechanism. In contrast, mesopores COFs (pore size > $2 \mathrm{~nm}$ ) result in high uptake pressure, large hysteresis, limited pore occupancy and low adsorption heat - these features lead to an unambiguous conclusion that large hydrophobic pores should be precluded for water confinement, uptake and exchange. COFs are unique in that their skeletons and pores are both predesignable. The present approach is not limited to the current skeletons but widely extendable to other microporous COFs with different building blocks, linkages and pore sizes to achieve water confinement. These COFs would be applicable in many areas, including water uptake, transport and separation as well as heatpump energy conversion.

\footnotetext{
Methods

Characterisations. Powder X-Ray diffraction (PXRD) patterns were recorded on Bruker D8 Advance by depositing powder on poly(methyl methacrylate) (PMMA) substrate, from $2^{\circ}$ to $30^{\circ}$ at with $0.02^{\circ}$ increment. Nitrogen sorption experiments were performed at $77 \mathrm{~K}$ on Micromeritics Instrument Corporation model 3Flex surface characterisation. The Brunauer-Emmett-Teller (BET) method was utilised to calculate the specific surface areas. By adopting the non-local density functional theory (NLDFT) model, the pore size distribution profile and pore volume were derived from the sorption curve. FT IR spectra were recorded on a Bruker Alpha with $\mathrm{KBr}$ disks. ${ }^{1} \mathrm{H}$ NMR spectra were recorded on a Bruker $500 \mathrm{MHz}$ NMR. Solid state ${ }^{13} \mathrm{C}$ pulse magic angle spinning nuclear magnetic resonance spectroscopy $\left({ }^{13} \mathrm{C}\right.$ CP/MAS NMR) spectra were recorded on a Bruker DNP-NMR $400 \mathrm{MHz}$. CHN Elemental analysis was recorded on a ThermoFisher Scientific FlashSmart CHNS Elemental Analyser by weighing the samples in tin containers and then combusted at high temperature with oxygen.
}

Computational studies. The monolayer structure of HFPTP-DMePDA-COF was constructed from the HFPTP and DMePDA building blocks using AuToGraFS and optimized using the self-consistent charge density functional tight binding (SCCDFTB) method and the mio-0-1 parameter set and Lennard-Jones dispersion. All calculations were undertaken using DFTB + version 1.3. Molecular modelling and Pawley refinement were carried out using a software package for crystal determination from PXRD pattern, implemented in Material Studio modelling version 8 (Accerlrys Inc.). We performed Pawley refinement to optimize the lattice parameters iteratively until the $R_{\mathrm{wp}}$ and $R_{\mathrm{p}}$ values converge. The pseudo-Voigt profile function for whole profile fitting and asymmetry correction function as BerarBaldinozzi were used during the refinement processes. The final unit cell parameters and refinement factors are $a=b=25.8092 \AA, c=4.5279 \AA, \alpha=\beta=90^{\circ}$, $\gamma=120^{\circ}, R_{\mathrm{wp}}=3.72 \%$ and $R_{\mathrm{p}}=2.15 \%$, respectively.

Vapour sorption. Vapour sorption experiment was performed on a Quantachrome Instruments model iQ3 equipped with manifold, vapour source and circulating water bath. Samples were degassed under vacuum at $120^{\circ} \mathrm{C}$ for $2 \mathrm{~h}$ prior to measurement. The water adsorption isotherms are performed at $283.15 \mathrm{~K}, 288.15 \mathrm{~K}$ and $298.15 \mathrm{~K}$ where the saturated vapour pressures were 9.2 torr, 12.8 torr and 23.8 torr respectively. The unit of measured volumetric vapour adsorption quantity was converted from $\mathrm{cm}^{3} \mathrm{~g}^{-1}$ to $\mathrm{g} \mathrm{g}^{-1}$ with an equation of $\mathrm{w}=(\mathrm{V} / 22414) \times 18.020$. The isosteric heat of adsorption $\left(Q_{\mathrm{st}}\right)$ was calculated with Clausius-Clapeyron equation $\triangle_{a d s} H_{w}=R\left(\frac{\partial \ln p}{\partial\left(\frac{1}{T}\right)}\right)_{W}$

Materials. Dichloromethane, hexane and tetrahydrofuran (THF, stabilized with BHT) were purchased from Avantor Performance Materials. $o$-Dichlorobenzene ( $o$-DCB), $n$-butanol ( $n$-BuOH), dioxane, mesitylene, trifluoromethanesulfonic acid, paraformaldehyde, hydrobromic acid $(41 \%)$, acetic acid and hydrazobenzene were purchased from TCI Chemicals. Toluene, methanol $(\mathrm{MeOH})$ and dimethylformamide (DMF) were purchased from VWR Chemicals. Potassium carbonate and potassium permanganate were purchased from Goodrich Chemical Enterprise. 1,3,6,8-Tetrabromopyrene, tetrakis(triphenylphosphine)palladium(0), 1,4-dimethoxybenzene, 4-aminobenzonitrile, 2,3,6,7,10,11-hexabromotriphenylene, 4-formylphenylboronic acid, 1,4-phenylenediamine (PDA), 2,5-dimethyl-1,4-phenylenediamine (DMePDA), 1,3,5-benzenetricarboxylic acid and $N$-hydroxysuccinimide (NBS) were purchased from BLDPharm. Carbazole, lithium aluminum hydride, pyridinium chlorochromate and $n$-butyllithium solution $(2.0 \mathrm{M}$ in cyclohexane) were purchased from Sigma Aldrich.

Synthesis. 2,3,6,7,10,11-Hexakis(4-formylphenyl) triphenylene (HFPTP) ${ }^{15}, 4,4^{\prime}, 4^{\prime \prime}-$ (1,3,5-triazine-2-4-6-triyl)trianiline (TTA $)^{30}, 1,3,5$-triformylbenzene (TFB) ${ }^{31}$, 3,3',6,6'-tetraformyl-9,9'-bicarbazole (TFBCz) ${ }^{19}, 1,3,6,8$-tetrakis (4-fomylphenyl) pyrene (TFPPy) ${ }^{21}, 1,1^{\prime}$-biphenyl-4,4'-diamine (BPDA) ${ }^{32}$ and 2,5-dimethoxyterephthalaldehyde (DMTP) $)^{33,34}$ were synthesized according to reported literatures.

HFPTP-PDA-COF ${ }^{\mathbf{1 4}}$. An $o$-DCB/ $n$ - $\mathrm{BuOH} / 2 \mathrm{M} \mathrm{AcOH}$ (4/6/1 by vol.; $1.1 \mathrm{~mL}$ ) mixture of HFPTP $(20 \mathrm{mg}, 0.028 \mathrm{mmol})$ and PDA $(14 \mathrm{mg}, 0.129 \mathrm{mmol})$ in a Pyrex tube $(10 \mathrm{~mL})$ was degassed by three freeze-pump-thaw cycles. The tube was sealed and heated at $120^{\circ} \mathrm{C}$ under vacuum for 7 days. The precipitate was collected by suction filtration and washed five times with THF and then Soxhlet with THF overnight. The powder was dried at $120^{\circ} \mathrm{C}$ under vacuum overnight to yield HFPTP-PDA-COF in an isolated yield of $85 \%$.

HFPTP-DMePDA-COF. An $o$-DCB/ $n$-BuOH/12 M AcOH (5/5/1 by vol.; $1.1 \mathrm{~mL})$ mixture of HFPTP $(15 \mathrm{mg}, 0.021 \mathrm{mmol})$ and DMePDA $(9.3 \mathrm{mg}, 0.068 \mathrm{mmol})$ in a Pyrex tube $(10 \mathrm{~mL})$ was degassed by three freeze-pump-thaw cycles. The tube was sealed and heated at $120^{\circ} \mathrm{C}$ under vacuum for 7 days. The precipitate was collected by suction filtration and washed five times with THF and then Soxhlet with THF overnight. The powder was dried at $120^{\circ} \mathrm{C}$ under vacuum overnight to yield HFPTP-DMePDA-COF in an isolated yield of $78 \%$.

HFPTP-BPDA-COF ${ }^{15}$. An $o$-DCB $/ n-\mathrm{BuOH} / 6 \mathrm{M} \mathrm{AcOH}(5 / 5 / 1$ by vol.; $1.1 \mathrm{~mL})$ mixture of HFPTP $(20 \mathrm{mg}, 0.028 \mathrm{mmol})$ and BPDA $(15.6 \mathrm{mg}, 0.084 \mathrm{mmol})$ in a Pyrex tube $(10 \mathrm{~mL})$ was degassed by three freeze-pump-thaw cycles. The tube was sealed and heated at $120^{\circ} \mathrm{C}$ under vacuum for 7 days. The precipitate was collected by suction filtration and washed five times with THF and then Soxhlet with THF overnight. The powder was dried at $120^{\circ} \mathrm{C}$ under vacuum overnight to yield HFPTP-BPDA-COF in an isolated yield of $77 \%$.

TFBCz-PDA-COF ${ }^{19}$. A 1,4-dioxane/mesitylene/6 $\mathrm{M} \mathrm{AcOH} \mathrm{(5/5/1} \mathrm{by} \mathrm{vol.;} 1.1 \mathrm{~mL}$ ) mixture of TFBCz $(37 \mathrm{mg}, 0.083 \mathrm{mmol})$ and PDA $(18 \mathrm{mg}, 0.17 \mathrm{mmol})$ in a Pyrex tube $(10 \mathrm{~mL})$ was degassed by three freeze-pump-thaw cycles. The tube was sealed and heated at $120^{\circ} \mathrm{C}$ under vacuum for 3 days. The precipitate was collected by suction filtration and washed five times with THF and then Soxhlet with THF overnight. The powder was dried at $120^{\circ} \mathrm{C}$ under vacuum overnight to yield TFBCz-PDA-COF in an isolated yield of $78 \%$. 
TFPPy-PDA-COF ${ }^{21}$. A 1,4-dioxane/6 M AcOH (10/1 by vol.; $1.1 \mathrm{~mL}$ ) mixture of TFPPy $(20 \mathrm{mg}, 0.032 \mathrm{mmol})$ and PDA $(7.7 \mathrm{mg}, 0.071 \mathrm{mmol})$ in a Pyrex tube $(10 \mathrm{~mL})$ was degassed by three freeze-pump-thaw cycles. The tube was sealed and heated at $120^{\circ} \mathrm{C}$ under vacuum for 3 days. The precipitate was collected by suction filtration and washed five times with THF and then Soxhlet with THF overnight. The powder was dried at $120^{\circ} \mathrm{C}$ under vacuum overnight to yield TFPPy-PDACOF in an isolated yield of $86 \%$.

TTA-TFB-COF ${ }^{20}$. An $o$-DCB $/ n$-BuOH/6 M AcOH (5/5/1 by vol.; $1.1 \mathrm{~mL}$ ) mixture of TTA $(35.4 \mathrm{mg}, 0.1 \mathrm{mmol})$ and TFB $(16.2 \mathrm{mg}, 0.1 \mathrm{mmol})$ in a Pyrex tube $(10 \mathrm{~mL})$ was degassed by three freeze-pump-thaw cycles. The tube was sealed and heated at $120^{\circ} \mathrm{C}$ under vacuum for 3 days. The precipitate was collected by suction filtration and washed five times with THF and then Soxhlet with THF overnight. The powder was dried at $120^{\circ} \mathrm{C}$ under vacuum overnight to yield TTA-TFB-COF in an isolated yield of $84 \%$.

TPB-DMTP-COF 22 . An $o-\mathrm{DCB} / n-\mathrm{BuOH} / 6 \mathrm{M} \mathrm{AcOH}(5 / 5 / 1$ by vol.; $1.1 \mathrm{~mL}$ ) mixture of TPB $(30 \mathrm{mg}, 0.076 \mathrm{mmol})$ and DMTP $(20.5 \mathrm{mg}, 0.15 \mathrm{mmol})$ in a Pyrex tube $(10 \mathrm{~mL})$ was degassed by three freeze-pump-thaw cycles. The tube was sealed and heated at $120^{\circ} \mathrm{C}$ under vacuum for 3 days. The precipitate was collected by suction filtration and washed five times with THF and then Soxhlet with THF overnight. The powder was dried at $120^{\circ} \mathrm{C}$ under vacuum overnight to yield TPB-DMTPCOF in an isolated yield of $81 \%$.

TTA-TFB-CMP. A MeCN/6 M AcOH (10/1 by vol.; $1.1 \mathrm{~mL}$ ) mixture of TTA $(35.4 \mathrm{mg}, 0.1 \mathrm{mmol})$ and TFB $(16.2 \mathrm{mg}, 0.1 \mathrm{mmol})$ in a Pyrex tube $(10 \mathrm{~mL})$ was degassed by three freeze-pump-thaw cycles. The tube was sealed and heated at $90^{\circ} \mathrm{C}$ under vacuum for 1 day. The precipitate was collected by suction filtration and washed five times with THF and then Soxhlet with THF overnight. The powder was dried at $120^{\circ} \mathrm{C}$ under vacuum overnight to yield TTA-TFB-CMP in an isolated yield of $82 \%$.

\section{Data availability}

The datasets generated during and/or analysed during the current study are available from the corresponding authors on request.

Received: 29 June 2021; Accepted: 19 October 2021;

Published online: 19 November 2021

\section{References}

1. Zhang, Y. S. \& Khademhosseini, A. Advances in engineering hydrogels. Science 356, eaaf3627 (2017).

2. O'Leary, L. E. R., Fallas, J. A., Bakota, E. L., Kang, M. K. \& Hartgerink, J. D. Multi-hierarchical self-assembly of a collagen mimetic peptide from triple helix to nanofibre and hydrogel. Nat. Chem. 3, 821-828 (2011).

3. Wylie, R. G. et al. Spatially controlled simultaneous patterning of multiple growth factors in three-dimensional hydrogels. Nat. Mater. 10, 799-806 (2011).

4. Henninger, S. K., Jeremias, F., Kummer, H. \& Janiak, C. MOFs for use in adsorption heat pump processes. Eur. J. Inorg. Chem. 2012, 2625-2634 (2012).

5. Canivet, J., Fateeva, A., Guo, Y., Coasne, B. \& Farrusseng, D. Water adsorption in MOFs: fundamentals and applications. Chem. Soc. Rev. 43, 5594-5617 (2014).

6. Burtch, N. C., Jasuja, H. \& Walton, K. S. Water stability and adsorption in metal-organic frameworks. Chem. Rev. 114, 10575-10612 (2014).

7. Sui, H., Han, B.-G., Lee, J. K., Walian, P. \& Jap, B. K. Structural basis of waterspecific transport through the AQP1 water channel. Nature 414, 872-878 (2001).

8. de Groot, B. L. \& Grubmüller, H. Water permeation across biological membranes: mechanism and dynamics of Aquaporin-1 and GlpF. Science 294, 2353-2357 (2001).

9. Leibler, L. Theory of microphase separation in block copolymers. Macromolecules 13, 1602-1617 (1980).

10. Matsen, M. W. \& Bates, F. S. Unifying weak- and strong-segregation block copolymer theories. Macromolecules 29, 1091-1098 (1996).

11. Radlauer, M. R. et al. Order and disorder in $\mathrm{ABCA}^{\prime}$ tetrablock terpolymers. J. Phys. Chem. B 124, 10266-10275 (2020).

12. Dalapati, S. et al. Rational design of crystalline supermicroporous covalent organic frameworks with triangular topologies. Nat. Commun. 6, 7786 (2015).

13. Li, J., Wang, J., Wu, Z., Tao, S. \& Jiang, D. Ultrafast and stable proton conduction in polybenzimidazole covalent organic frameworks via confinement and activation. Angew. Chem. Int. Ed. 60, 12918-12923 (2021).

14. $\mathrm{Xu}, \mathrm{S}$. et al. Luminescent $\mathrm{sp}^{2}$-carbon-linked $2 \mathrm{D}$ conjugated polymers with high photostability. Chem. Mater. 32, 7985-7991 (2020).

15. Wang, P. et al. High-precision size recognition and separation in synthetic 1D nanochannels. Angew. Chem. Int. Ed. 58, 15922-15927 (2019).

16. Ng, E.-P. \& Mintova, S. Nanoporous materials with enhanced hydrophilicity and high water sorption capacity. Microporous Mesoporous Mater. 114, 1-26 (2008).

17. Pires, J., Pinto, M., Estella, J. \& Echeverría, J. C. Characterization of the hydrophobicity of mesoporous silicas and clays with silica pillars by water adsorption and DRIFT. J. Colloid Interface Sci. 317, 206-213 (2008).

18. Björklund, S. \& Kocherbitov, V. Alcohols react with MCM-41 at room temperature and chemically modify mesoporous silica. Sci. Rep. 7, 9960 (2017).

19. Feng, S. et al. Bicarbazole-based redox-active covalent organic frameworks for ultrahigh-performance energy storage. Chem. Commun. 53, 11334-11337 (2017).

20. Wang, P. et al. Exceptional iodine capture in $2 \mathrm{D}$ covalent organic frameworks. Adv. Mater. 30, 1801991 (2018)

21. Rabbani, M. G. et al. A 2D mesoporous imine-linked covalent organic framework for high pressure gas storage applications. Chem. Eur. J. 19, 3324-3328 (2013)

22. Xu, H., Gao, J. \& Jiang, D. Stable, crystalline, porous, covalent organic frameworks as a platform for chiral organocatalysts. Nat. Chem. 7, 905-912 (2015).

23. Liu, X., Wang, X. \& Kapteijn, F. Water and metal-organic frameworks: from interaction toward utilisation. Chem. Rev. 120, 8303-8377 (2020).

24. Inagaki, S., Fukushima, Y., Kuroda, K. \& Kuroda, K. Adsorption isotherm of water vapor and its large hysteresis on highly ordered mesoporous silica. $J$. Colloid Interface Sci. 180, 623-624 (1996).

25. Hwang, J., Kataoka, S., Endo, A. \& Daiguji, H. Adsorption and desorption of water in two-dimensional hexagonal mesoporous silica with different pore dimensions. J. Phys. Chem. C. 119, 26171-26182 (2015).

26. Nguyen, H. L. et al. A porous covalent organic framework with voided square grid topology for atmospheric water harvesting. J. Am. Chem. Soc. 142, 2218-2221 (2020)

27. Biswal, B. P. et al. Pore surface engineering in porous, chemically stable covalent organic frameworks for water adsorption. J. Mater. Chem. A 3, 23664-23669 (2015).

28. Tao, S. et al. Confining $\mathrm{H}_{3} \mathrm{PO}_{4}$ network in covalent organic frameworks enables proton super flow. Nat. Commun. 11, 1981 (2020).

29. Low, J. J. et al. Virtual high throughput screening confirmed experimentally: porous coordination polymer hydration. J. Am. Chem. Soc. 131, 15834-15842 (2009).

30. Gomes, R., Bhanja, P. \& Bhaumik, A. A triazine-based covalent organic polymer for efficient $\mathrm{CO}_{2}$ adsorption. Chem. Commun. 51, 10050-10053 (2015).

31. Li, Z. et al. A $2 \mathrm{D}$ azine-linked covalent organic framework for gas storage applications. Chem. Commun. 50, 13825-13828 (2014).

32. Clegg, J. K. et al. A stimuli responsive system of self-assembled anion-binding $\mathrm{Fe}_{4} \mathrm{~L}_{68}{ }^{+}$cages. Chem. Sci. 4, 68-76 (2013).

33. Palmgren, A., Thorarensen, A. \& Bäckvall, J.-E. Efficient synthesis of symmetrical 2,5-disubstituted benzoquinones via palladium-catalysed double Negishi coupling. J. Org. Chem. 63, 3764-3768 (1998).

34. Kuhnert, N., Rossignolo, G. M. \& Lopez-Periago, A. The synthesis of trianglimines: on the scope and limitations of the $[3+3]$ cyclocondensation reaction between $(1 R, 2 R)$-diaminocyclohexane and aromatic dicarboxaldehydes. Org. Biomol. Chem. 1, 1157-1170 (2003).

\section{Acknowledgements}

D.J. acknowledges supports by the Singapore MOE tier 1 grant (R-143-000-C06-114), MOE tier 1 grant (R-143-000-C05-114) and MOE tier 2 grant (MOE-T2EP10220-0004).

\section{Author contributions}

D.J. conceived the project, designed experiments and provided funds. K.T. S.T. and N.H conducted the experiments. D.J. and K.T. wrote the manuscript and discussed the results with all authors. All data are reported in the main text and supplementary materials.

\section{Competing interests}

The authors declare no competing interests. 


\section{Additional information}

Supplementary information The online version contains supplementary material available at https://doi.org/10.1038/s41467-021-27128-4.

Correspondence and requests for materials should be addressed to Donglin Jiang.

Peer review information Nature Communications thanks the anonymous reviewers for their contribution to the peer review of this work. Peer reviewer reports are available.

Reprints and permission information is available at http://www.nature.com/reprints

Publisher's note Springer Nature remains neutral with regard to jurisdictional claims in published maps and institutional affiliations. (c) (P) Open Access This article is licensed under a Creative Commons Attribution 4.0 International License, which permits use, sharing, adaptation, distribution and reproduction in any medium or format, as long as you give appropriate credit to the original author(s) and the source, provide a link to the Creative Commons license, and indicate if changes were made. The images or other third party material in this article are included in the article's Creative Commons license, unless indicated otherwise in a credit line to the material. If material is not included in the article's Creative Commons license and your intended use is not permitted by statutory regulation or exceeds the permitted use, you will need to obtain permission directly from the copyright holder. To view a copy of this license, visit http://creativecommons.org/ licenses/by/4.0/.

(C) The Author(s) 2021 